\title{
Retención de videocápsula endoscópica, una nueva enfermedad médico-quirúrgica: serie de casos
}

\author{
Endoscopic video capsule retention, a new medical surgical disease: case series
}

\author{
Luis F. Cabrera-Vargas ${ }^{1,2}$, Ricardo Villarreal ${ }^{1,2,3}$, Jean Pulido-Segura ${ }^{1,4 *}$, Mauricio Pedraza-Ciro ${ }^{1,2}$, \\ Sebastián Sánchez-Ussa ${ }^{1,5}$ y Laura T. Padilla-Pinzón ${ }^{4,6}$ \\ ${ }^{1}$ Departamento Cirugía General, Universidad El Bosque; ${ }^{2}$ Departamento de Cirugía General, Los Cobos Medical Center; ${ }^{3}$ Departamento de Cirugía \\ Gastrointestinal y Endoscopia, Los Cobos Medical Center; ${ }^{4}$ Departamento de Medicina General, Universidad el Bosque; ${ }^{5}$ Departamento Cirugía \\ General, Pontificia Universidad Javeriana; ${ }^{6}$ Departamento de Cirugía Pediátrica, Hospital de la Misericordia. Bogotá, Colombia
}

\begin{abstract}
Resumen
Antecedentes: La videocápsula endoscópica (VCE), método mínimamente invasivo de estudio del sistema gastrointestinal indicado en hemorragia digestiva oculta, tiene como principal complicación la retención de la cápsula endoscópica (RCE). Actualmente se maneja con extracción directa empleando enteroscopia de doble balón; sin embargo, cuando falla por su localización o por patologías asociadas, la intervención quirúrgica resulta el método de mayor éxito. Objetivo: Evaluar las características clínicas y el manejo de pacientes con RCE. Método: De enero de 2001 a diciembre de 2018 se realizó un búsqueda retrospectiva, en tres centros, de pacientes con diagnóstico de RCE. Se evaluaron variables clínicas y de manejo, y además se realizó una revisión crítica de la literatura. Resultados: Se hallaron 15 pacientes con diagnóstico de RCE (ocho mujeres y siete hombres), con una edad promedio de 65 años (rango: 45-77). Siete requirieron manejo quirúrgico, tres manejo endoscópico y cinco manejo médico. Conclusiones: La RCE es una patología nueva de baja prevalencia. El manejo quirúrgico resulta ser una segunda línea terapéutica cuando la extracción de la VCE no ha sido posible endoscópicamente. Para su diagnóstico y manejo, esta patología requiere una especial atención a la historia clínica, los antecedentes y los síntomas de los pacientes.
\end{abstract}

Palabras clave: Hemorragia digestiva de origen oscuro. Laparotomía. Obstrucción intestinal. Patency capsule. Retención de cápsula endoscópica. Videocápsula endoscópica.

\begin{abstract}
Background: The endoscopic video capsule (EVC), a minimally invasive method of study of the gastrointestinal system indicated in occult gastrointestinal bleeding, has as main complication the retention of endoscopic capsule (CR), currently it is managed with direct extraction using double-balloon enteroscopy; however, when it fails due to its location or associated pathologies, the surgical intervention results in the most successful approach. Objective: To evaluate clinical characteristics and management of patients with video capsule endoscopy retention. Method: From January 2001 to December 2018, a retrospective search was carried out in three centers, of patients with $C R$ diagnosis, clinical variables and approach were evaluated, in addition to a critical review of the literature. Results: 15 patients with a diagnosis of CR (eight 8 women and seven men), average age 65 (range: 45-77). Seven required surgical management, three endoscopic and five medical management.
\end{abstract}

\footnotetext{
Correspondencia:

*Jean Pulido-Segura

Ak. $9,131 \mathrm{a}-20$

Universidad El Bosque

C.P. 110121

Fecha de recepción: 30-09-2019

Bogotá, Colombia

E-mail: japulido@unbosque.edu.co

DOI: $10.24875 /$ CIRU.20001614

Cir Cir. 2020;88(6):714-720

Contents available at PubMed www.cirugiaycirujanos.com

0009-7411/@ 2020 Academia Mexicana de Cirugía. Publicado por Permanyer. Este es un artículo open access bajo la licencia CC BY-NC-ND (http://creativecommons.org/licenses/by-nc-nd/4.0/).
} 
Conclusions: The CR is a new pathology of low prevalence, the surgical management resulting from a second therapeutic line when the removal of the VCE has not been possible endoscopically, this pathology requires special attention in the patient's medical history and symptoms for diagnosis and management.

Key words: Dark digestive hemorrhage. Laparotomy. Intestinal obstruction. Patency capsule. Endoscopic capsule retention. Video endoscopic capsule.

\section{Introducción}

La videocápsula endoscópica (VCE) se ha convertido en la herramienta de mayor utilidad para el estudio del intestino delgado, permitiendo su visualización directa con una mejor tolerancia ${ }^{1-5}$. La VCE se utiliza clásicamente en el estudio de sangrado digestivo oculto, pero en la última década se ha demostrado su utilidad en el diagnóstico y el seguimiento de otro tipo de enfermedades, como la enfermedad de Crohn, ante la sospecha de tumores de intestino delgado, y en el diagnóstico y el seguimiento del síndrome de poliposis hereditario ${ }^{2,6-9}$.

Este procedimiento no está exento de complicaciones, siendo la retención de la cápsula endoscópica (RCE) la principal, con una incidencia menor del $2 \%$, que requiere generalmente manejo médico, endoscópico o quirúrgico para su extracción $n^{3,10-12}$.

En la actualidad, la RCE es manejada con extracción directa empleando enteroscopia de doble balón; sin embargo, si el paciente presenta signos de obstrucción intestinal mecánica de alto grado o sangrado gastrointestinal, la intervención quirúrgica debe tomarse como una herramienta que permite no solo la extracción de la VCE, sino también el diagnóstico y el tratamiento de la causa de la retención en un solo tiempo ${ }^{13-16}$.

Nos permitimos presentar nuestra experiencia en el manejo de RCE en tres instituciones, con una serie de 15 casos, junto con un análisis crítico con revisión de la literatura.

\section{Método}

Se realizó una búsqueda retrospectiva de enero de 2010 a julio de 2018 en tres centros de Bogotá, Colombia, de pacientes con diagnóstico de RCE. Se evaluaron la patología por la cual se encontraba retenida la VCE y las características clínicas y sociodemográficas, dentro de las cuales se tuvieron en cuenta la edad, el sexo, la indicación de VCE, el tiempo de retención, la clínica al momento de la valoración (dolor abdominal, irritación peritoneal, obstrucción intestinal), el método de extracción (médico, endoscópico, abierto, laparoscópico), el tiempo quirúrgico, la causa de la retención, los días de hospitalización, el sangrado, el requerimiento de unidad de cuidado intensivo, las complicaciones y la mortalidad.

Se planteó con los pacientes encontrados un posible abordaje desde el manejo médico instaurado, endoscópico o quirúrgico, que requieren.

El protocolo de estudio fue aprobado por el comité de ética de las tres instituciones e implementado de conformidad con las disposiciones de la Declaración de Helsinki y las guías de buena práctica clínica.

\section{Análisis estadístico}

Al ser una cohorte retrospectiva observacional, se realizó un análisis descriptivo de los datos, prospectivamente en las bases de datos de Microsoft Excel y utilizando SPSS1 v. 22.0. Las variables continuas fueron analizadas por rangos. Las variables se resumen utilizando mediana, valores mínimo y máximo, y porcentajes.

\section{Resultados}

En los tres centros se encontraron 15 pacientes que presentaron RCE, cuyas características sociodemográficas se presentan en la tabla 1. La edad promedio de los pacientes fue de 65.5 años (rango: 45-86). El $53.3 \%$ fueron de sexo femenino y el $46.7 \%$ de sexo masculino. El tiempo promedio de retención de la cápsula fue de 2.6 semanas. El $33.3 \%$ se resolvió con manejo médico, el $20.0 \%$ fue llevado a manejo endoscópico y el $46.6 \%$ requirió manejo quirúrgico.

Todos los pacientes fueron llevados a VCE por sangrado de vías digestivas oculto, definido por doble estudio endoscópico de vías digestivas altas y dos colonoscopias sin hallazgos concluyentes para el sangrado.

Las causas de la retención fueron enfermedad neoplásica (tres neoplasias a nivel de la válvula ileocecal y un tumor del estroma gastrointestinal), enfermedad de Crohn (tres), enteritis por radiación (tres), síndrome 
Tabla 1. Distribución demográfica de la población

\begin{tabular}{lc}
\hline Variables & $\mathbf{n}(\%)$ \\
\hline Sexo & $7(47)$ \\
Masculino & $8(53)$ \\
Femenino & \\
Indicación & $15(100)$ \\
Estudio de hemorragia digestiva intermedia u oscura & \\
Dolor abdominal & \\
Sí & $9(60)$ \\
No & $6(40)$ \\
Causa de retención & \\
Neoplasia (válvula ileocecal) & \\
Enfermedad de Crohn & $4(30)$ \\
Enteritis por radiación & $3(20)$ \\
Síndrome de Peutz-Jeghers & $3(20)$ \\
Divertículo de Meckel & $2(13)$ \\
Enteropatía por antiinflamatorios no esteroideos & $1(6.6)$ \\
Estenosis posoperatoria & $1(6.6)$ \\
Manejo definitivo & $1(6.6)$ \\
Médico & \\
Endoscópico & \\
Laparotomía & $5(33.3)$ \\
\hline
\end{tabular}

de Peutz-Jeghers (dos), divertículo de Meckel (uno), enteropatía por antiinflamatorios no esteroideos (uno) y estenosis posoperatoria (uno).

Tres pacientes con RCE fueron diagnosticados de enfermedad de Crohn de novo, y en dos de ellos la RCE se resolvió con manejo médico empleando corticoides; el otro paciente presentaba estenosis y fibrosis en un segmento de íleon proximal, por lo cual para resolver la RCE requirió manejo quirúrgico con enterotomía y plastia intestinal de la estenosis.

Los pacientes con RCE secundaria a neoplasias presentaron lesiones a nivel de la válvula ileocecal y del íleon terminal, y al no tener respuesta con el manejo médico debido a la RCE mecánica generada por la masa se llevaron a manejo quirúrgico.

En el caso del paciente con hallazgo de enteropatía por antiinflamatorios no esteroideos (debida a consumo crónico, de más o menos 6 meses), en el estudio de sangrado oculto se decidió ampliar los estudios con una VCE degradable, pero como en nuestro medio no se cuenta con esta tecnología se usó una VCE convencional y presentó RCE, la cual se resolvió médicamente (Tabla 2).

Dentro del grupo de los pacientes con manejo quirúrgico, en el $100 \%$ fue abierto debido a la gran distensión de asas que impidieron el abordaje laparoscópico. El promedio de tiempo quirúrgico fue de 94.2 minutos, el promedio de sangrado fue de 90
Tabla 2. Tipo de manejo según diagnóstico por retención de cápsula endoscópica

\begin{tabular}{lcccc}
\hline $\begin{array}{l}\text { Causas con } \\
\text { manejo }\end{array}$ & $\mathbf{n}(\%)$ & Laparotomía & Endoscopia & Médico \\
\hline Neoplasia & $4(26)$ & 4 & 0 & 0 \\
$\begin{array}{l}\text { Enfermedad de } \\
\text { Crohn }\end{array}$ & $3(20)$ & 1 & 0 & 2 \\
$\begin{array}{l}\text { Enteritis por } \\
\text { radiación }\end{array}$ & $3(20)$ & 1 & 0 & 2 \\
$\begin{array}{l}\text { Síndrome de } \\
\text { Peutz-Jeghers }\end{array}$ & $2(13)$ & 0 & 2 & 0 \\
$\begin{array}{l}\text { Divertículo de } \\
\text { Meckel }\end{array}$ & $1(6.6)$ & 1 & 0 & 0 \\
$\begin{array}{l}\text { Enteropatía por } \\
\text { antiinflamatorios no }\end{array}$ & $1(6.6)$ & 0 & 0 & 1 \\
esteroideos & & & 1 & 0 \\
$\begin{array}{l}\text { Estenosis } \\
\text { posoperatoria }\end{array}$ & $1(6.6)$ & 0 & & \\
\hline
\end{tabular}

cc y el promedio de hospitalización fue de 10.8 días. Solo se reportaron dos complicaciones posoperatorias: una infección del sitio operatorio de grado II y una neumonía adquirida en la comunidad.

Ningún paciente requirió manejo en la unidad de cuidado intensivo, todos resolvieron su sintomatología tras la realización del procedimiento quirúrgico, no hubo mortalidad y el seguimiento se dio a 6 meses sin complicaciones.

\section{Discusión}

El Dr. Paul Swain y el ingeniero Gabriel Iddan, tras 20 años de investigación, lograron para el año 2001 la aprobación por parte de la Food and Drug Administration de los Estados Unidos de la VCE ${ }^{1,5,12,13}$, una cápsula de $11 \times 33 \mathrm{~mm}$ que logró integrar una cámara en miniatura, una fuente de luz, baterías y un transmisor de radio ${ }^{17}$. Desde entonces, la VCE se ha vuelto la técnica de referencia en la evaluación completa del intestino delgado ${ }^{5,7,12}$, ya que presentó avances para el paciente por ser un método diagnóstico fisiológico que disminuye las incomodidades a su paso por el tracto gastrointestinal y que poco o nada altera la cotidianidad del paciente, al no requerir sedación ni suspensión de anticoagulación y no limitar la actividad física ${ }^{17,18}$.

El procedimiento se inicia con un ayuno nocturno, seguido de la ingesta de 2 litros de polietilenglicol 
durante 2 horas, iniciando su consumo 4 horas antes de ingerir la cápsula 6 ,13,14,16. Se debe dar orden estricta al paciente (oral y escrita) de estar pendiente de las heces para revisión de la salida de la cápsula, para informar posteriormente al médico tratante ${ }^{6,7}$. Se espera que la VCE se elimine en la deposición entre 12 y 72 horas después de su ingestión ${ }^{2,9}$. Sin embargo, hay un periodo de 14 días para su salida ${ }^{18}$, después del cual se habla de RCE (Fig. 1) y se deben tomar medidas médicas, endoscópicas o quirúrgicas para su retiro ${ }^{3,9,10}$. La obtención de imágenes del ciego es un indicador positivo debido a los bajos niveles de obstrucción producidos en el colon ${ }^{7}$. El estudio en el que no es posible durante el tiempo de grabación visualizar la primera imagen cecal se conoce como estudio incompleto, aunque esto no necesariamente implica retención ${ }^{18}$. Entre los factores asociados a estudio incompleto se encuentran: tiempo prolongado de tránsito gástrico, gastroparesia, diabetes mellitus, pobre preparación intestinal, hipertiroidismo, cirugía intestinal previa, pacientes hospitalizados y en especial los postrados en cama y en la unidad de cuidado intensivo ${ }^{11,14}$. Un estudio realizado por Stanich, et al. ${ }^{14}$ evidenció la relación que tiene el incremento de la actividad física con un menor tiempo de tránsito intestinal, pero no se demuestra una asociación con la disminución de los casos de estudios incompletos.

A pesar de ser la más frecuente complicación, la RCE tiene una baja incidencia, con registros que varían en la literatura entre el 0.3 y el $2 \% \%^{1,2,7}$. Estas cifras no se han modificado en los últimos 10 años $^{7}$, y aún no hay un método estandarizado para la prevención de la retención ${ }^{6,10,17}$. Un estudio realizado por Han, et al. ${ }^{6}$ utilizando la cápsula OMOM o Miro Cam recogió un total de 5348 pacientes, de los cuales a 77 se les diagnosticó RCE tras 14 días de no expulsión y una radiografía que evidenciaba la presencia de la cápsula en el abdomen. Se sospecha la RCE en pacientes asintomáticos en quienes no hay evidencia de expulsión en los siguientes 14 días tras la ingestión de la VCE y en pacientes que desarrollan clínica de obstrucción con o sin perforación, como es el caso del $40 \%$ de nuestros pacientes, y que además no presentan pruebas de excreción de la cápsula ${ }^{6,7,13}$.

En los pacientes asintomáticos, la radiografía es la herramienta de preferencia para confirmar la RCE. Aunque el paciente tenga implantes, como marcapasos, raramente estos impiden que una radiografía evidencie la ubicación de la cápsula, incluso cuando esta se ha desbaratado $0^{7,15}$. La tomografía computada ha demostrado un mejor rendimiento, ya que permite especificar la

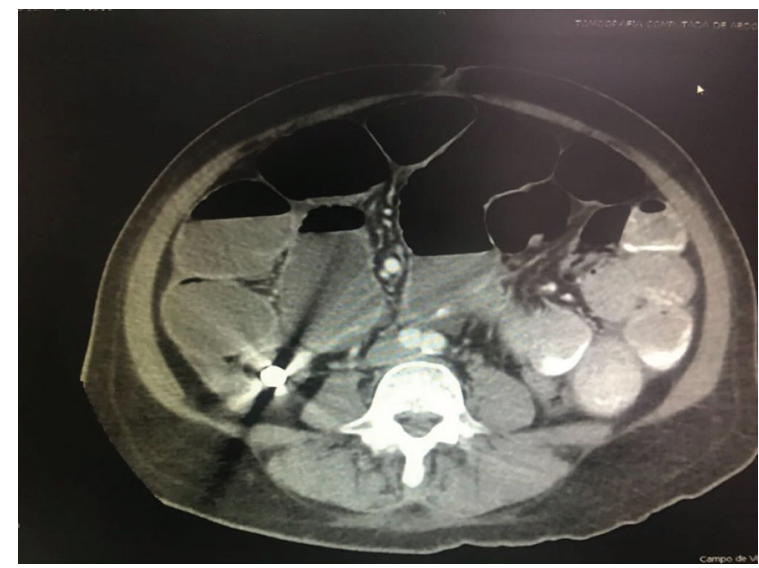

Figura 1. Laparotomía exploratoria en la que se evidencia retención de videocápsula endoscópica en el íleon distal secundaria a presencia de masa en la válvula ileocecal.

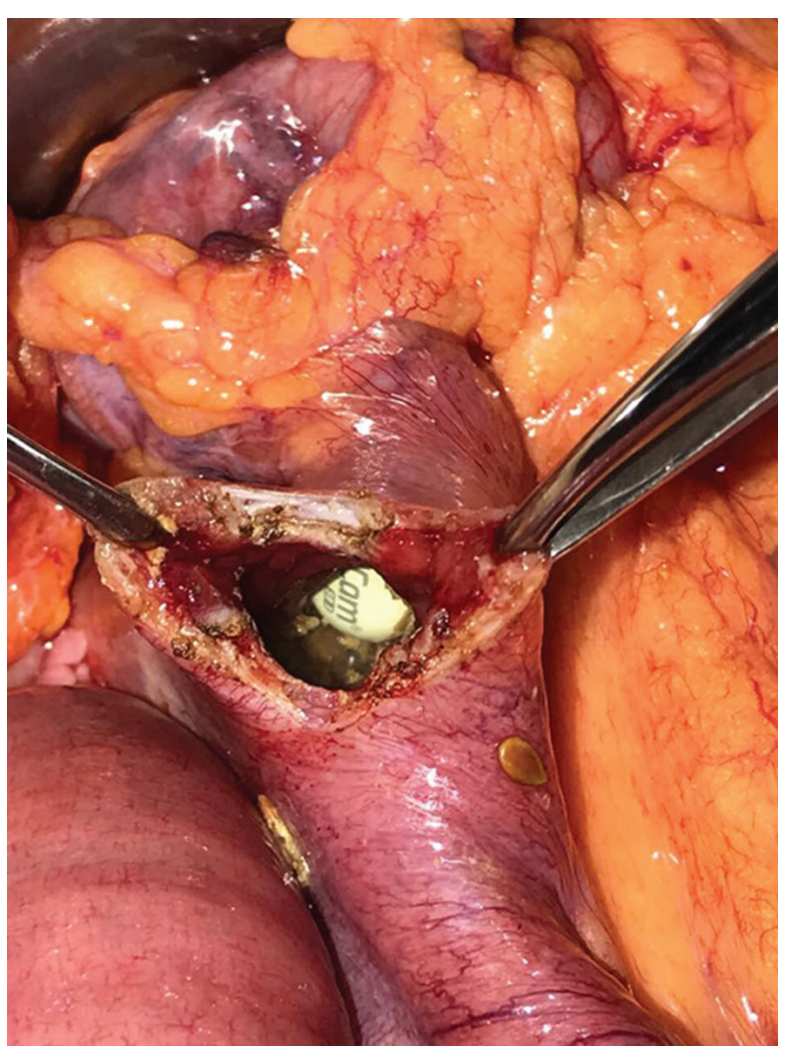

Figura 2. Tomografía computada abdominal contrastada en la que se evidencia una obstrucción intestinal de alto grado con retención de videocápsula endoscópica a nivel del íleon distal. No identifica una causa clara de la retención.

ubicación de la cápsula en el tracto gastrointestinal e identificar una posible causa de la retención, como en nuestra serie de pacientes ${ }^{7}$ (Fig. 2). Se han evidenciado casos en que la clínica de obstrucción aguda se presenta incluso años después de la ingestión de la VCE ${ }^{2,7}$. 
Una alternativa que ha mostrado buenos resultados para evitar la incidencia de RCE es el uso de la Patency capsule (PC), una cápsula biodegradable que se degrada más o menos a las 30 horas de su ingestión ${ }^{1}$ y que evita para el paciente la exposición a radiación. Es relativamente económica y puede repetirse en caso de persistir dudas. De esta forma se puede advertir la posible obstrucción por VCE con una especificidad del $83 \%$ y una sensibilidad del $97 \%$, llegando al $100 \%$ cuando se usan imágenes de cortes seccionados asociados a la $\mathrm{PC}^{7,9}$. A pesar de sus beneficios, aún no se encuentra indicado su uso sistemático ${ }^{1}$, siendo la enfermedad inflamatoria intestinal (EII) la principal indicación de la PC ${ }^{9}$.

Las indicaciones de la VCE son muy específicas. Entre ellas encontramos la hemorragia digestiva de origen oscuro (oculta, manifiesta o anemia ferropénica) como la más frecuente, con cifras que oscilan entre el 60 y el $70 \%$ de los pacientes. Sin embargo, en nuestro estudio, el $100 \%$ de los pacientes tuvo esta indicación. Esta situación se define como una hemorragia que persiste o recurre tras las realización de una gastroscopia y una colonoscopia con resultado negativo (por sangrado intermitente o error humano) ${ }^{10,13}$. Después de la hemorragia digestiva de origen oscuro se encuentran las enfermedades inflamatorias intestinales, como la enfermedad de Crohn no estenosante, los tumores del intestino delgado sin sospecha de estenosis (que representan solo un 3-6\% de todos los tumores del tracto gastrointestinal, presentándose con sintomatología de hemorragia digestiva de origen oscuro, dolor abdominal, náuseas, vómitos, pérdida de peso y anorexia) ${ }^{1}$, los síndromes de poliposis hereditaria (poliposis adenomatosa familiar, síndrome de Peutz-Jeghers) y la enfermedad celíaca, lo cual se correlaciona con los hallazgos en nuestra serie $^{13-16,18}$.

En pacientes con hemorragia digestiva de origen oscuro, un estudio con VCE negativo tiene tasas bajas de resangrado, del $4.6 \%$, comparado con el $48 \%$ de resangrado en pacientes con hallazgos positivos en la VCE ${ }^{12,13,18}$. Se ha encontrado una mayor prevalencia en menores de 40 años con tumores del intestino delgado, divertículos de Meckel, lesión de Dieulafoy y enfermedad de Crohn', y en mayores de 40 años con lesiones vasculares y lesiones secundarias a antiinflamatorios no esteroideos ${ }^{13-15}$.

Es importante aclarar que no se ha encontrado asociación del tamaño de la cápsula con la RCE ni con la edad de los pacientes, pero sí se ha visto una estrecha relación con las indicaciones clínicas por las que se realiza el procedimiento; llega a ser de un $13 \%$ en pacientes con riesgo de Ell, y se encuentran también diferencias entre si se indica al paciente con diagnóstico o con suposición de Ell (5-13\% contra $1.4-3.5 \%)^{7,9,18}$. Sin embargo, no son las cifras más altas registradas, pues se encontraron cifras del 10$20 \%$ en pacientes sometidos a VCE por obstrucción del intestino delgado subaguda y hasta del $25 \%$ en pacientes con tumores del intestino delgado. Los porcentajes más bajos de RCE (2\%) se encontraron en pacientes a quienes se indicó por sospecha de sangrado del intestino delgado, diarrea crónica o dolor abdominal. Estos datos sugieren que la Ell puede ser considerada un factor de riesgo de RCE, así como lo son la resección previa de intestino delgado, la terapia de radiación abdominal y el uso crónico de antiinflamatorios no esteroideos, lo que refuerza la importancia de una buena anamnesis del paciente que será sometido a tratamiento con $\mathrm{VCE}^{4,5,7}$. También son contraindicaciones los síntomas obstructivos, una ileocolonoscopia que evidencie estenosis, la evidencia radiológica o la sospecha de estenosis en el tracto gastrointestinal y el seguimiento de tumores del intestino delgado ${ }^{11-13}$.

Se ha demostrado que el método primario para el tratamiento de la RCE es la enteroscopia de doble balón con o sin asistencia por dispositivo, con un éxito del 56 al 100\% en la recuperación de la cápsula ${ }^{2,6}$, previo estudio de localización. Se indica enteroscopia de doble balón a pacientes que muestren dolor abdominal asociado a RCE, aunque en pacientes asintomáticos con RCE el tratamiento médico específico puede mantenerse hasta que la cápsula pase espontáneamente o hasta que los síntomas de obstrucción aparezcan, teniendo en cuenta que del 35 al $50 \%$ de los pacientes excretan la cápsula después de 15 días sin ninguna terapia, como reportan Han, et al. ${ }^{6}$ en su un estudio de 77 pacientes con RCE, de los cuales 16 resolvieron el cuadro sin intervención.

Por la cantidad de pacientes con clínica positiva de obstrucción intestinal, se opta primero por la realización de tomografía computada y luego procedimiento quirúrgico si el paciente no resuelve su cuadro obstructivo. Obligatoriamente debe ser no solo para recuperar la cápsula, sino como tratamiento de la causa de la obstrucción ${ }^{7,10}$, como se realizó en los diferentes casos expuestos. Nemeth, et al. ${ }^{3}$ realizaron un estudio con 2401 pacientes, de los que 25 presentaron RCE y ocho recibieron manejo quirúrgico, aunque solo en dos la cirugía se realizó de urgencia dada la instauración de obstrucción intestinal; al igual que en la 
totalidad de nuestros pacientes, esta intervención eliminó la sintomatología tras su realización.

El manejo quirúrgico puede dilatarse. Baichi, et al. ${ }^{19}$ mostraron en su serie de pacientes que en patologías oncológicas debe realizarse el procedimiento a necesidad, teniendo en cuenta la posible instauración de síntomas por RCE.

Como se mencionó anteriormente, el cuadro de obstrucción se ha evidenciado incluso años después del procedimiento con VCE, tal como muestran Han, et al. ${ }^{6}$ en su estudio, en el que cerca del $65 \%$ de los pacientes con RCE requirieron intervención quirúrgica dentro de los 5 años siguientes. La extracción de la VCE tras el diagnóstico de RCE se ha realizado principalmente por cirugía, siendo este el método de recuperación más utilizado (45-60\%). Se logra el paso espontáneo en el 3 al 15.8\%, y finalmente un abordaje endoscópico en el 7 al $12.5 \%$, que para nuestra serie correspondió al 20\%,10,20. El $46.6 \%$ de nuestros pacientes fueron llevados a manejo quirúrgico, de modo similar a la serie de Lee, et al. ${ }^{20}$, quienes demostraron por análisis multivariado que ante la instauración de sintomatología obstructiva por RCE los pacientes fueron llevados a manejo quirúrgico abierto, incluso requerido en pacientes en quienes falla el manejo endoscópico o este no se encuentra disponible ${ }^{20}$.

Teniendo en cuenta la morbimortalidad que produce a futuro cualquier intervención quirúrgica, se han buscado alternativas de tratamiento médico que permitan evitar manejos invasivos. Han, et al. ${ }^{6}$ trataron con infliximab a 20 pacientes con enfermedad de Crohn y $\mathrm{RCE}$, y evidenciaron que el $80 \%$ resolvieron la estenosis y presentaron expulsión espontánea de la VCE. Rondonotti demostró que los pacientes con enfermedades inflamatorias del intestino delgado se benefician del tratamiento con esteroides, ya que hasta un 20-30\% presentaron expulsión de la VCE. Sin embargo, en estudios posteriores se demostró que los esteroides y los inmunosupresores son menos efectivos en la eliminación de la VCE que el tratamiento quirúrgico y el infliximab, ya que de 35 pacientes con enfermedad de Crohn que recibieron esteroides 0 inmunosupresores (sin infliximab) solo nueve no requirieron manejo quirúrgico ${ }^{6}$. Llama la atención la ausencia de resolución por vía laparoscópica, teniendo en cuenta los beneficios demostrados de este método.

La expulsión espontánea o la instauración de la obstrucción aguda se da en 4-12 semanas después de la ingestión en pacientes con $\mathrm{RCE}^{7}$. Se han determinado unos tiempos de recuperación de la cápsula razonables entre 3 y 6 meses en pacientes asintomáticos,

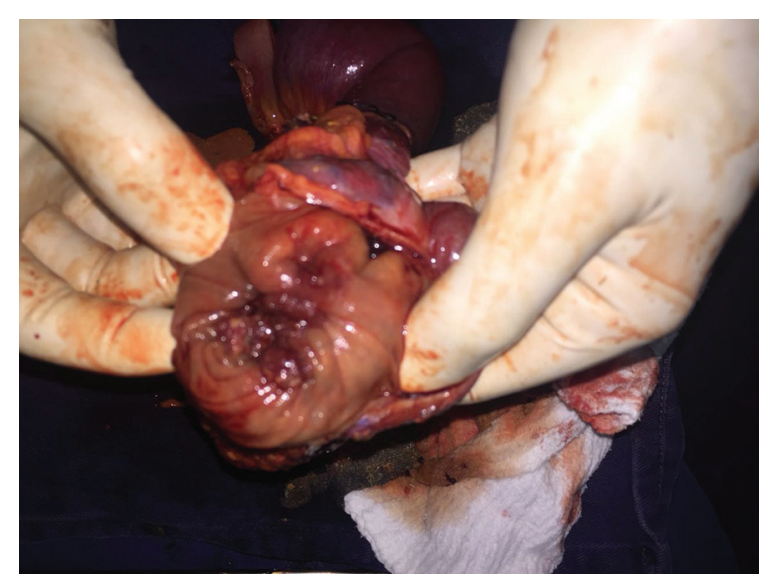

Figura 3. Pieza quirúrgica de masa en la válvula ileocecal que estenosa la luz del tracto gastrointestinal y genera la retención de la videocápsula endoscópica.

informando sobre los riesgos de tener la cápsula en su interior, y opciones de recuperación quirúrgica o endoscópica. Sin embargo, la intervención quirúrgica sigue siendo la primera opción en todos los casos en que las herramientas de diagnóstico sugieren inequívocamente la presencia de una enfermedad neoplásica, tal como reportan Han, et al. ${ }^{6}$, encontrando que la necesidad de un procedimiento quirúrgico se debía sobre todo a obstrucción intestinal, en un $62 \%$ de los casos, sospecha de malignidad en un $12 \%$, persistencia de sangrado del intestino delgado en un $12 \%$ y estenosis fibrótica grave identificada por endoscopia de doble balón en un 6\%. En nuestra serie de casos, la enfermedad neoplásica (Fig. 3) es la principal causa de retención, y la enfermedad de Crohn se da en igual número que la enteritis por radiación.

Teniendo en cuenta la revisión de la literatura y la serie de casos descrita, sugerimos un algoritmo de manejo de los pacientes con signos y síntomas de obstrucción intestinal asociado a RCE (Fig. 4).

La RCE es una patología poco frecuente, secundaria a un procedimiento diagnóstico endoscópico, y en general se resuelve de forma espontánea o empleando enteroscopia de doble balón. Sin embargo, puede requerir manejo quirúrgico ante la presencia de obstrucción intestinal de alto grado y sangrado gastrointestinal, con el fin de extraer de forma efectiva la VCE y tratar al mismo tiempo la causa de la retención.

\section{Conflicto de intereses}

Los autores declaran no tener conflictos de intereses, económicos, profesionales ni personales. 


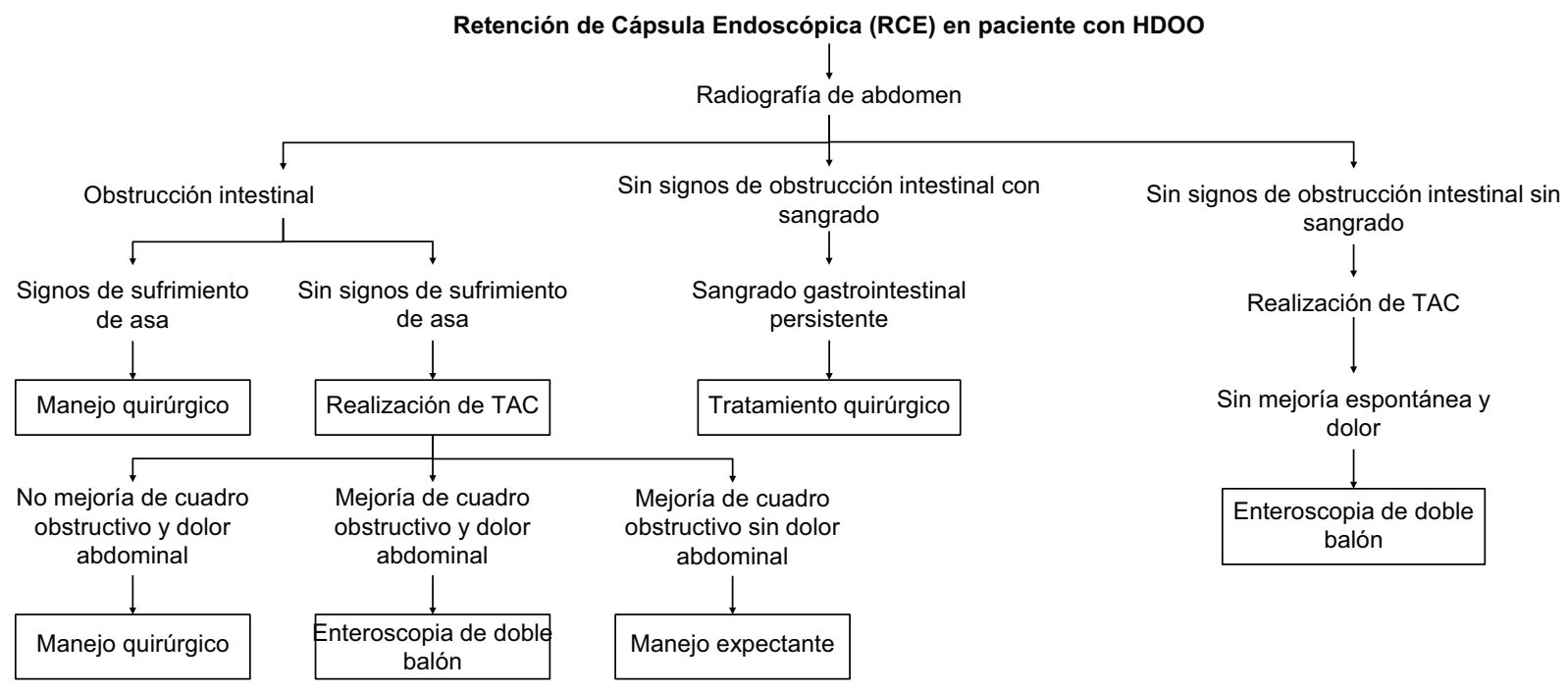

Figura 4. Algoritmo de manejo para pacientes que ingresan al servicio de urgencias con signos y síntomas de obstrucción intestinal asociada a retención de cápsula endoscópica.

\section{Financiamiento}

Los autores declaran no haber recibido ningún financiamiento de terceros.

\section{Responsabilidades éticas}

Protección de personas y animales. Los autores declaran que para esta investigación no se han realizado experimentos en seres humanos ni en animales.

Confidencialidad de los datos. Los autores declaran que han seguido los protocolos de su centro de trabajo sobre la publicación de datos de pacientes.

Derecho a la privacidad y consentimiento informado. Los autores han obtenido el consentimiento informado de los pacientes y/o sujetos referidos en el artículo. Este documento obra en poder del autor de correspondencia.

\section{Bibliografía}

1. Fernández MI, López AC, García SN. Protocolo de indicaciones de la videocápsula endoscópica. Medicine - Programa de Formación Médica Continuada Acreditado. 2020;13(1):59-62.

2. Sanhueza E, Ibáñez P, Araya R, Delgado I, Quezada S, Jadue L, et al. Utilidad de la cápsula endoscópica como método diagnóstico en el estudio de patología de ID. Rev Med Chile. 2010;138(3):303-8.

3. Nemeth A, Wurm Johansson G, Nielsen J, Thorlacius H, Toth E. Capsule retention related to small bowel capsule endoscopy: a large European single-center 10-year clinical experience. United European Gastroenterol J. 2017;5:677-86.

4. Goldstein JL, Eisen GM, Lewis B, Gralnek IM, Zlotnick S, Fort JG. Video capsule endoscopy to prospectively assess small bowel injury with cele- coxib, naproxen plus omeprazole, and placebo. Clin Gastroenterol Hepatol. 2005;3(2):133-41.

5. Kopylov U, Seidman EG. Role of capsule endoscopy in inflammatory bowel disease. World J Gastroenterol. 2014;20(5):1155-64.

6. Han Z, Qiao W, Ai X, Aimin L, Chen Z, Zhang J, et al. Risk factors for surgery in patients with retention of endoscopic capsule. Scand J Gastroenterol. 2018;53:107-13.

7. Rondonotti E. Capsule retention: prevention, diagnosis and management. Ann Transl Med. 2017;5(9):198.

8. Toth E, Marthinsen L, Bergström M, Park PO, Mansson P, Nemeth A, et al. Colonic obstruction caused by video capsule entrapment in a metal stent. Ann Transl Med. 2017:5:199.

9. Rezapour M, Amadi C, Gerson LB. Retention associated with video capsule endoscopy: systematic review and meta-analysis. Gastrointest Endosc. 2017;85(6):1157-68.e2.

10. Cheon JH, Kim YS, Lee IS, Chang DK, Ryu JK, Lee KJ, et al Can we predict spontaneous capsule passage after retention? A nationwide study to evaluate the incidence and clinical outcomes of capsule retention. Endoscopy. 2007;39:1046-52.

11. Pham T, Miller A, La Paglia D, Cham A. Small bowel obstruction with perforation secondary to PillCam. Case Rep Gastrointest Med. 2018;2018:9081742.

12. Rondonotti E, Soncini M, Girelli C, Ballardini G, Bianchi G, Brunati S, et al. Small bowel capsule endoscopy in clinical practice: a multicenter 7-year survey. Eur J Gastroenterol Hepatol. 2010;22:1380-6.

13. Van de Bruaene C, De Looze D, Hindryckx P. Small bowel capsule endoscopy: where are we after almost 15 years of use? World J Gastrointest Endosc. 2015;7:13-36.

14. Stanich PP, Peck J, Murphy C, Porter K, Meyer M. Physical activity during video capsule endoscopy correlates with shorter bowel transit time. Endosc Int Open. 2017;5:E856-60.

15. Bandorski D, Kurniawan N, Baltes P, Hoeltgen R, Hecker M, Stunder D, et al. Contraindications for video capsule endoscopy. World J Gastroenterol. 2016;22(45):9898-908

16. Shim KN, Jeon SR, Jang HJ, Kim J, Lim YJ, Kim KO, et al. Quality indicators for small bowel capsule endoscopy. Clin Endosc. 2017:50:148-60.

17. Appleyard M, Glukhovsky A, Swain P. Wireless-capsule diagnostic endoscopy for recurrent small-bowel bleeding. N Engl J Med. 2001;344:232-3.

18. Cedrón-Cheng H. Cápsula endoscópica del intestino delgado. Rev Gastroenterol Perú. 2010;30:341-9.

19. Baichi MM, Arifuddin RM, Mantry PS. Small-bowel masses found and missed on capsule endoscopy for obscure bleeding. Scand J Gastroenterol. 2007:42:1127-32

20. Lee HS, Lim YJ, Kim KO, Jang HJ, Chun J, Jeon SR, et al. Outcomes and management strategies for capsule retention: a Korean Capsule Endoscopy Nationwide Database Registry Study. Dig Dis Sci. 2019;64:3240-6. 